\title{
COVID-19 PANDEMIC IN DELUSIONS: TWO CASES
}

\author{
Ozgecan Tuna ${ }^{1}$, Serdar Salman ${ }^{2} \&$ Aslı Enez Darcin ${ }^{3}$ \\ ${ }^{I}$ Kanuni SS Training and Research Hospital, Department of Psychiatry, Istanbul, Turkey \\ ${ }^{2}$ Bakirkoy Training and Research Hospital for Psychiatry, Neurology and Neurosurgery, Department of Psychiatry, \\ Istanbul, Turkey \\ ${ }^{3}$ Istanbul Basaksehir Cam ve Sakura City Hospital, Department of Psychiatry, Istanbul, Turkey
}

received: 23.9.2020;

revised: 17.11.2020;

accepted: 1.12 .2020

$* * * * *$

\section{INTRODUCTION}

COVID-19 pandemic resulted in an increased social distress and previous experiences have shown that outbreaks are associated with a risk of relapse in individuals with severe mental illness, such as schizophrenia, schizoaffective disorder or bipolar disorder. Risky behaviors, noncompliance with treatment, substance abuse and anxiety levels may remarkably increase, and these may adversely affect the prognosis (Huremović 2019). Anxiety and depression levels in the general population were found to be increased during the COVID-19 outbreak and based on this finding potential impact of pandemic on psychosis was investigated (Özdin \& Bayrak Özdin 2020). Even though frequency of such symptoms were found to be low, a significant number of patients who have developed coronavirus-related psychotic symptoms were reported (Brown et al. 2020).

This case report aims to present and discuss two cases with delusions related to COVID-19 pandemic. The first case was tested negative for COVID-19 by Polymerase Chain Reaction (PCR) test and low-dose chest Computerized Tomography (CT), however he developed bizarre delusions in which he had transmitted COVID-19 to other people and spread the disease to the whole world. The second case was tested positive for COVID-19 by PCR test and she had delusions about others trying to harm her and her family by infecting them with coronavirus.

\section{CASE REPORTS}

$1^{\text {st }}$ Case

"My entire family died, because they contracted COVID-19 from me..."

A 34-year-old male refugee was brought to the emergency department with a self-inflicted knife injury during the second month of the COVID-19 pandemic. He was admitted to surgery ward for further treatment.

On mental status examination, patient explained that he is the source of COVID-19 and disease has spread everywhere, and people got sick because of him. He said he confirmed this fact by talking to God and when people learnt this fact, they murdered his family. It was understood that his thought content started to change by mid-April (2020), and he started to experience auditory hallucinations two weeks after that. The voices told him that he can prevent the spread of the virus if he kills himself and people would be saved. Then he attempted suicide by stabbing himself. He said he was getting the messages from the TV and he thought his family members were murdered because of him.

During his psychiatric examination, he had persecutory delusions such as others would harm him and the rest of his family since he was the source of COVID-19. Additionally, he had paranoid and reference delusions such as people saying that he is transmitting the disease and he has to die because of this. Also, he had bizarre delusions of communicating with God. He had auditory hallucinations commanding him to kill himself.

His total PANSS score was $161 / 210$ on admission. He was diagnosed with "schizophreniform disorder" according to Diagnostic and Statistical Manual of Mental Disorders $5^{\text {th }}$ Edition (DSM 5) criteria. This was his first psychiatric diagnosis. Routine laboratory and imaging studies were normal. No other pathologies were detected.

Olanzapine $10 \mathrm{mg} /$ day was administered and gradually increased to $20 \mathrm{mg}$ /day. Lorazepam $1 \mathrm{mg}$ /day was added to treatment for intermittent agitated behavior. His psychotic symptoms improved following treatment. His total PANSS score was 68/210 after 2 weeks of hospitalization.

\section{$2^{\text {nd }}$ Case}

"People are trying to infect me with coronavirus..."

A 52-year-old female patient was admitted to the emergency department following her attempt to jump out of the balcony of her house during the second month of the COVID-19 pandemic. Her attempt was stopped by her relatives. Her attempt was influenced by auditory hallucinations and those voices were commanding her to kill herself. Initial plan was to admit her to the psychiatry clinic due to refusal of treatment and selfdestruction risk, but findings on her low dose chest CT was compatible with COVID-19 pneumonia and she got admitted to the COVID-19 positive psychiatry ward. 
During her mental evaluation, it was reported that her paranoid thoughts started about a month ago, she was worried about other people harming her and her family by infecting them with coronavirus and her grandchildren dying because of this.

During her psychiatric examination she had persecutory delusions such as people would harm her by infecting with coronavirus and she had auditory hallucinations commanding her to kill herself.

Result of her nasopharyngeal swab sample PCR test was positive for COVID-19.

Her neuroimaging was reviewed by a neurologist and no organic pathology can be found.

Her total PANSS score was $159 / 210$ on admission. She was diagnosed with "schizophreniform disorder" according to DSM-5 criteria. This was her first psychiatric diagnosis.

She was administered hydroxychloroquine 800 $\mathrm{mg}$ /day perorally and enoxaparin sodium $0.8 \mathrm{ml} /$ day subcutaneously for 5 days for COVID-19. She was administered haloperidol $10 \mathrm{mg} /$ day and biperiden $5 \mathrm{mg}$ /day parenterally for psychiatric treatment. A partial improvement was observed in the severity of her psychotic and COVID-19 related symptoms during follow-up. Her total PANSS score after 1 week of hospitalization was 114/210.

Both patients did not have a history of any other psychiatric presentations or chronic conditions. They did not smoke nor used alcohol or any psychoactive substances, and no family history of mental illness was elicited.

\section{DISCUSSION}

We presented two noteworthy cases in the context of COVID-19 pandemic since their thought content included delusions constructed around the outbreak. It is a well-known fact that current events occasionally affect psychotic thought content and to our knowledge there are no previous case reports in the literature reporting onset of first psychotic symptoms during the COVID-19 outbreak with intense delusions of COVID-19 related content resulting suicide attempts. Current literature report cases with psychotic symptoms after or on-going treatment of COVID-19 (Finucane et al. 2000, Fischer et al. 2020).

Additionally, it is thought-provoking that in both cases first psychiatric symptoms were triggered by the COVID-19 outbreak and resulted in suicide attempts. Mental health consequences of infectious disease outbreaks were extensively studied and exacerbation in psychotic symptoms and increased the disease severity and suicidal ideas were reported (Huremović 2019). As COVID-19 pandemic emerges, patients developed psychotic symptoms and suicidal ideas were reported by other authors as well (Huarcaya-Victoria et al. 2020, Finatti et al. 2020).
Severe self-destructive behaviors in both of our cases supported the previously reported increasing phenomenon of self-destruction during outbreaks (Huremović 2019, Reger et al. 2020). It was speculated that in patients with delusions associated with the outbreak; some factors related to pandemic may have possibly caused their delusions to persist and increased their severity which lead to self-destructive actions as a result.

Our first case was a refugee in a foreign country. During epidemics, refugees are disadvantaged due to language problems, poor living conditions, challenges in accessing healthcare and they carry a higher fear and stigmatization risk (Truman et al. 2009, Person et al. 2004). We believe such factors might have affected our case, since his delusions involved feelings of guilt and severe fear and he was thinking that he could transmit COVID-19 to others.

Our learnings from these cases show that there may be an increase in cases with psychosis during pandemics as well as exacerbation of psychotic symptoms. Also, it should be remembered that in addition to the significant increase in the frequency and severity of psychiatric conditions, patients may experience a first psychotic episode during a pandemic similar to our cases presented in this report and external reality can impact the content of their delusions.

\section{Acknowledgements: None.}

\section{Conflict of interest: None to declare.}

\section{Contribution of individual authors:}

Özgecan Tuna: examined the patient, reviewed the literature, wrote the first and last version of the manuscript.

Serdar Salman: examined the patient, collected the clinical data.

Asli Enez Darcin: examined the patient, reviewed the evidence and contributed to all the versions of the manuscript.

\section{References}

1. Brown E, Gray R, Lo Monaco S, O'Donoghue B, Nelson B, Thompson A et al.: The potential impact of COVID-19 on psychosis: A rapid review of contemporary epidemic and pandemic research. Schizophr Res 2020; S09209964(20)30257-7

2. Finatti F, Pigato G, Pavan $C$, Toffanin $T \&$ Favaro A: Psychosis in Patients in COVID-19-Related Quarantine: A Case Series. Prim Care Companion CNS Disord 2020; 22:20102640

3. Finucane ML, Alhakami A, Slovic P \& Johnson SM: The affect heuristic in judgments of risks and benefits. J Behav Decis Making 2000; 13:1-17

4. Fischer M, Coogan AN, Faltraco F \& Thome J: COVID19 paranoia in a patient suffering from schizophrenic psychosis-a case report. Psychiatry Res 2020; 288:113001 
5. Huarcaya-Victoria J, Herrera D \& Castillo C: Psychosis in a patient with anxiety related to COVID-19: A case report. Psychiatry Res 2020; 289:113052

6. Huremović D: Preparing for the Outbreak. In: Huremović D. (eds) Psychiatry of Pandemics, 65-77. Springer Cham, 2019

7. Özdin $S \&$ \&ayrak Özdin Ş: Levels and predictors of anxiety, depression and health anxiety during COVID-19 pandemic in Turkish society: The importance of gender. Int J Soc Psychiatry 2020; 66:504-511
8. Person B, Sy F, Holton K, Govert B, Liang A, Garza B et al.: Fear and Stigma: The Epidemic within the SARS Outbreak. Emerging Infectious Diseases 2000; 10:358-363

9. Reger MA, Stanley IH \& Joiner TE: Suicide Mortality and Coronavirus Disease 2019-A Perfect Storm? JAMA Psychiatry 2020

10. Truman B, Tinker T, Vaughan E, Kapella B, Brenden M, Woznica $C$ et al.: Pandemic Influenza Preparedness and Response Among Immigrants and Refugees. American Journal Of Public Health 2009; 99(Suppl 2):S278-S286

Correspondence:

Ozgecan Tuna, MD

Kanuni SS Training and Research Hospital, Department of Psychiatry

Turgut Ozal Bulvari No 1, Kucukcekmece, Istanbul, Turkey

E-mail: ozgecantuna@gmail.com 